\title{
Methods to Obtain Basic Probability Assignment in Evidence Theory
}

\author{
Tazid Ali \\ Dept. of Mathematics \\ Dibrugarh University \\ Dibrugarh-786004, India
}

\author{
Palash Dutta \\ Dept. of Mathematics \\ Dibrugarh University \\ Dibrugarh-786004, India
}

\begin{abstract}
Dempster-Shafer Theory (DST) of Evidence is a powerful and flexible mathematical tool for handling uncertainty, impreciseness, and incomplete information. It can be used when both epistemic and aleatory uncertainties are present in the problem under consideration. The fundamental and important object of this theory of evidence is the primitive function called basic probability assignment (bpa). In the absence of empirical data, experts in related fields provide necessary information (bpa). However how to obtain BPA is still an open issue. In this paper, we propose methods to determine BPA when only the minimum, maximum and most likely values of the parameter are known. An example is illustrated to demonstrate and check the efficiency of the proposed methods. We have also developed an extended version of uncertainty measurement in evidence theory in order to calculated total uncertainty in the body of evidence obtained by the proposed methods.
\end{abstract}

Keywords: Evidence Theory, Basic Probability Assignment, Fuzzy Set

\section{INTRODUCTION}

Modelling real problems typically involves processing uncertainty of three types. Uncertainty due to randomness (Aleotary uncertainty), uncertainty due to lack of specification and uncertainty due to ambiguity about set boundaries (fuzziness). Traditionally probability theory is used to deal with aleatory uncertainty and fuzzy theory takes care of fuzziness. Dempster put forward a theory and now it is known as evidence theory or Dempster- Shafer theory (1976). This theory is nowadays widely uses for the epistemic and aleatory uncertainty analysis. The use of Dempster-Shafer theory in risk analysis has many advantages over the conventional probabilistic approach. It provides convenient and comprehensive way to handle engineering problems including, imprecisely specified distributions, poorly known and unknown correlation between different variables, modelling uncertainty, small sample size, and measurement uncertainty. The fundamental and important object of this theory of evidence is the primitive function called basic probability assignment (bpa). But how to obtain basic probability is still open issue. Various researchers tried to address this problem using different methods. S. P. Kurkowska et al. 2000 [4] calculated BPA in Diagnosis support considering actual population. Z. Zuo et al 2009 [6] proposed a method of rough set theory based on random set and BP neural network to obtain BPA. Z. Yibing et al, 2010 [5] developed method to assign BPA based on fuzzy subordinate. W. Jiang et al, 2011 [7] proposed a new method to obtain BPA based on distance measure between the sample data under test and model of attribute of species. In this paper, we also propose three methods of assigning BPA when only three values of the parameter are known, viz. minimum, maximum and most likely value.

\section{BASIC CONCEPT OF FUZZY SET THEORY}

Environmental/human health risk assessment is an important aid in any decision-making process in order to minimize the effects of human activities on the environment. Unfortunately, usually environmental data tends to be vague and imprecise, so uncertainty is associated with any study related with these kinds of data. Fuzzy set theory provides a way to characterize the imprecisely defined variables, define relationships between variables based on expert human knowledge and use them to compute results. In this section, some necessary backgrounds and notions of fuzzy set theory [3] that will be required in the sequel are reviewed.

Definition 2.1 Let $X$ be a universal set. Then the fuzzy subset A of $X$ is defined by its membership function $\mu_{A}: X \rightarrow[0,1]$

Which assign a real number $\mu_{A}(x)$ in the interval $[0,1]$, to each element $x \in A$, where the value of $\mu_{A}(x)$ at $\mathrm{x}$ shows the grade of membership of $\mathrm{x}$ in A.

Definition 2.2 Given a fuzzy set $A$ in $X$ and any real number $\boldsymbol{\alpha} \in[0,1]$. Then the $\boldsymbol{\alpha}$-cut or $\boldsymbol{\alpha}$-level or cut worthy set of A, denoted by ${ }^{\alpha} \mathrm{A}$ is the crisp set

$$
{ }^{\alpha} A=\left\{x \in X: \mu_{A}(x) \geq \alpha\right\}
$$

The strong a cut, denoted by ${ }^{\alpha+} \mathrm{A}$ is the crisp set

$$
{ }^{\alpha} A=\left\{x \in X: \mu_{A}(x)>\alpha\right\}
$$

Definition 2.3 The support of a fuzzy set A defined on $\mathrm{X}$ is a crisp set defined as

$$
\operatorname{Supp}(\mathrm{A})=\left\{x \in X: \mu_{A}(x)>0\right\}
$$

Definition 2.4 The height of a fuzzy set A, denoted by $h(A)$ is the largest membership grade obtain by any element in the set and it is denoted as

$$
h(A)=\sup _{x \in X} \mu_{A}(x)
$$

Definition 2.5 A fuzzy number is a convex normalized fuzzy set of the real line $\mathrm{R}$ whose membership function is piecewise continuous. 
Definition 2.6 A triangular fuzzy number A can be defined as a triplet $[a, b, c]$. Its membership function is defined as:

$$
\mu_{A}(x)=\left\{\begin{array}{l}
\frac{x-a}{b-a}, a \leq x \leq b \\
\frac{c-x}{c-b}, b \leq x \leq c
\end{array}\right.
$$

Definition 2.7 A trapezoidal fuzzy number A can be expressed as [a, b, c, d] and its membership fuzzy number is defined as:

$$
\mu_{A}(x)=\left\{\begin{array}{l}
\frac{x-a}{b-a}, a \leq x \leq b \\
1, b \leq x \leq c \\
\frac{d-x}{d-c}, c \leq x \leq d
\end{array}\right.
$$

\section{BASIC CONCEPTS OF DEMPSTER- SHAFER THEORY OF EVIDENCE}

Evidence theory [2] is one of the important tools to handle both aleatory and epistemic uncertainty. The use of DempsterShafer theory in risk analysis has many advantages over the conventional probabilistic approach. It provides convenient and comprehensive way to handle engineering problems including, imprecisely specified distributions, poorly known and unknown correlation between different variables, modelling uncertainty, small sample size, and measurement uncertainty.

A frame of discernment (or simply a frame), usually denoted as $\Theta$ is a set of mutually exclusive and exhaustive propositional hypotheses, one and only one of which is true. Evidence theory is based on two dual non-additive measure, i.e. belief measure and plausible measure. There is one important function in Dempster-Shafer theory to define belief measure and plausible measure which is known as basic probability assignment (bpa).

A function $m: 2^{\Theta} \rightarrow[0,1]$ is called basic probability assignment (bpa) on the set $\Theta$ if it satisfies the following two conditions:

$$
\begin{gathered}
m(\phi)=0 \\
\sum_{A \subseteq \Theta} m(A)=1
\end{gathered}
$$

Where $\phi$ is an empty set and $\mathrm{A}$ is any subset of $\Theta$.

The Basic Probability Assignment function (or mass function) is a primitive function. Given a frame, $\Theta$, for each source of evidence, a mass function assigns a mass to every subset of $\Theta$, which represents the degree of belief that one of the hypotheses in the subset is true, given the source of evidence.

The subset $A$ of frame $\Theta$ is called the focal element of $m$, if $m(A)>0$.

Using the basic probability assignment (bpa), belief measure and plausibility measure are respectively determined as

$$
\operatorname{Bel}(A)=\sum_{B \subseteq A} m(B), A \subseteq \Theta \text { and } P l(A)=\sum_{B \cap A \neq \Phi} m(B)
$$

Here $m(B)$ is the degree of evidence in the set $B$ alone, whereas $\operatorname{Bel}(A)$ is the total evidence in set $A$ and all subset $B$ of $A$ and the plausibility of an event $\mathrm{A}$ is the total evidence in set $A$, plus the evidence in all sets of the universe that intersect with $A$. Where $\operatorname{Bel}(A)$ and $\operatorname{Pl}(A)$ represent the lower bound and upper bound of belief in $A$. Hence, interval $[\operatorname{Bel}(A)$, $P l(A)]$ is the range of belief in $A$.

Given two mass functions $m_{1}$ and $m_{2}$, Dempster-Shafer theory also provides Dempster's combination rule for combining them, which is defined as follows:

$$
m(C)=\frac{\sum_{A \cap B=C} m_{1}(A) m_{2}(B)}{1-\sum_{A \cap B=\phi} m_{1}(A) m_{2}(B)}
$$

\section{UNCERTAINTY QUANTIFICATION}

The concept [1] of information is intimately connected with the concept of uncertainty. The most fundamental aspects of this connection is that uncertainty involved in any problem solving situation is a result of some information deficiency. Information (pertaining to the model within which the situation is conceptualized) may be incomplete, imprecise, fragmentary, not fully reliable, vague, contradictory, or deficient in other ways. In general, these various information deficiencies may result in different types of uncertainty.

Uncertainty based information was first conceive in terms of classical set theory and, later, in terms of probability theory. In addition to classical set theory and probability theory, uncertainty based information is now well understood in fuzzy set theory, possibility theory and evidence theory.

Three types of uncertainty are now recognized in the five theories, in which measurement of uncertainty is currently well established. They are: nonspecificity (or imprecision), which is connected with sizes (cardinalities) of relevant sets of alternatives; fuzziness (or vagueness), which results from imprecise boundary of fuzzy sets; and strife (or discord), which expresses conflicts among the various sets of alternatives. In this section we briefly explain the different uncertainty measure.

\subsection{Uncertainty in Crisp Set}

\subsubsection{Nonspecificity of crisp sets:}

Measurement of uncertainty (and associated information) was first conceived in terms of classical set theory. It was shown by Hartley (1928) that using a function form the class of function

$$
U(A)=c \cdot \log _{b}|A|,
$$

Where $|A|$ denotes the cardinality of a finite nonempty set $A$, and $\mathrm{b}, \mathrm{c}$ are positive constant $(\mathrm{b}>1, \mathrm{c}>0)$, is the only sensible way to measure the amount of uncertainty associated with a finite set of possible alternatives. Each choice of values of the constant $\mathrm{b}$ and $\mathrm{c}$ determines the unit in which uncertainty is measured. When $b=2$ and $c=1$, which is the most common choice, uncertainty is measured in bits, and we get

$$
U(A)=\log _{2}|A|
$$

The function $U$ is called Hartley function. The form (1) is applicable only for finite sets. However, this form may be approximately modified to infinite sets on $\boldsymbol{R}$. Given a 
measurable and Lebesgue-integral subset A of R, the corresponding Hartley function is given by

$$
U(A)=\log [1+\mu(A)]
$$

where $\mu(A)$ is the measure of A defined by the Lebesgue integral of the characteristic function of A. For instance, when $\mathrm{A}$ is an interval $[\mathrm{a}, \mathrm{b}]$ on $\mathrm{R}$, then $\mu(\mathrm{A})=\mathrm{b}-\mathrm{a}$ and

$$
U(A)=\log [1+b-a]
$$

\subsection{Uncertainty in Fuzzy Set Theory}

\subsubsection{Nonspecificity of Fuzzy Sets:}

A natural generalization of the Hartley function from classical set theory to fuzzy set theory was proposed in the early 1980s under the name $U$-uncertainty. For any nonempty fuzzy set A defined on a finite universal set $\mathrm{X}$, the generalized Hartley function has the form:

$$
U(A)=\left.\frac{1}{h(A)} \int_{0}^{h(A)} \log _{2}\right|^{\alpha} A \mid d \alpha
$$

Where $\left.\right|^{\alpha} A \mid$ denotes the cardinality of the $\alpha$-cut of $\mathrm{A}$ and $h(A)$ is the height of A.

When a nonempty fuzzy set A is defined on $\boldsymbol{R}$, and the $\alpha$-cuts of ${ }^{\alpha} A$ are infinite sets, we have to calculate $U(A)$ by modified the form

$$
U(A)=\frac{1}{h(A)} \int_{0}^{h(A)} \log _{2}\left[1+\mu\left({ }^{\alpha} A\right)\right] d \alpha
$$

which is a generalization of (2). It is assume that ${ }^{\alpha} A$ is a measurable and Lebesgue-integral function; $\mu\left({ }^{\alpha} A\right)$ is the measure of ${ }^{\alpha} A$ defined by the Lebesgue integral of the characteristic function of ${ }^{\alpha} A$. As for continuous fuzzy set $\alpha$ cuts produces close interval i.e., ${ }^{\alpha} A=\left[A_{1}(\alpha), A_{2}(\alpha)\right]$, then $\mu\left({ }^{\alpha} A\right.$ )$=\left[A_{2}(\alpha)-A_{l}(\alpha)\right]$. So, (4) becomes

$$
U(A)=\frac{1}{h(A)} \int_{0}^{h(A)} \log _{2}\left[1+A_{2}(\alpha)-A_{1}(\alpha)\right] d \alpha
$$

\subsubsection{Fuzziness of fuzzy sets:}

Other than nonspecificity, fuzzy sets have one more form of uncertainty and that is fuzziness.

There are different ways of measuring fuzziness but the following is the most frequently used. For a fuzzy set A, defined on an universal set $X$, the fuzziness $f(A)$ is given by

$$
f(\mathrm{~A})=\Sigma\{1-|2 \mathrm{~A}(\mathrm{x})-1|: \mathrm{x} \in \mathrm{X}\} .
$$

If $X$ is some interval of $R$, say $X=[a, b]$, then the above formula is replaced by

$$
f(A)=\int_{a}^{b}(1-|2 A(x)-1|) d x f(\mathrm{~A})
$$

\subsection{Uncertainty in Evidence Theory:}

In evidence theory two types of uncertainty, nonspecificity and strife coexist and both are measured in the same units.

\subsubsection{Nonspecificity}

The Hartley function, as a measure of nonspecificity, was first generalized from classical set theory, fuzzy set theory and possibility theory. Once this generalization function, the $U$ uncertainty, was well established in possibility theory, a special branch of evidence theory, it was relatively easy to generalized it further and make it applicable within all of evidence theory.

For a body of evidence $(\mathfrak{I}, \mathrm{m})$, the nonspecificity is defined by the formula

$$
N(m)=\sum_{A \in \mathfrak{I}} m(A) \log _{2}|A|
$$

\subsubsection{Strife}

The another uncertainty, strife, of evidence theory is defined by the formula

$$
S(m)=-\sum_{A \in \mathfrak{I}} m(A) \log _{2} \sum_{B \in \mathfrak{I}} m(B) \frac{|A \cap B|}{|A|}
$$

Since the two types of uncertainty, nonspecificity and strife, coexist in evidence theory, and both are measured in the same units, it is reasonable to consider the possibility of adding their individual measure to form a measure of total uncertainty. The total uncertainty is defined by the formula:

$$
\begin{aligned}
N S(m) & =N(m)+S(m) \\
& =\sum_{A \in \mathfrak{I}} m(A) \log _{2}|A|-\sum_{A \in \mathfrak{I}} m(A) \log _{2} \sum_{B \in \mathfrak{I}} m(B) \frac{|A \cap B|}{|A|} \\
& =\sum_{A \in \mathfrak{I}} m(A) \log _{2} \frac{|A|^{2}}{\sum_{B \in \mathfrak{I}} m(B)|A \cap B|}
\end{aligned}
$$

This is applicable only when the focal elements of the body of evidence are finite. We have proposed an approximate modification of (8) to be applicable for infinite case. In the proposed modification $|\mathrm{A}|$ is replaced by $[1+\mu(\mathrm{A})]$, where $\mu(A)$ is the measure of A defined by the Lebesgue integral of the characteristic function of A. So the total uncertainty is given by

$$
N S(m)=\sum_{A \in \mathfrak{I}} m(A) \log _{2} \frac{[1+\mu(A)]^{2}}{\sum_{B \in \mathfrak{I}} m(B)[1+\mu(A \cap B)]}
$$

In particular for $A=[1, b] \mu(A)=b-a$. So (9) takes the form

$$
N S(m)=\sum_{A \in \mathfrak{I}} m(A) \log _{2} \frac{[1+b-a]^{2}}{\sum_{B \in \mathfrak{I}} m(B)[1+d-c]}
$$

In the sequel we will be using the form (10) for calculation of uncertainty for a body of evidence.

\section{Proposed Methods to obtain BPA}

In this section we proposed three new methods of bpa assignment when only information about the parameter is the minimum, maximum and most likely value. Generally in such a situation, the parameter is expressed a triangular fuzzy number with [min, $\max ]$ as support and the most likely value as core. Then there is a standard procedure of obtaining focal elements from a fuzzy number and assigning bpas. For that $n$ 
number of equally spaced $\alpha$-cuts are considered which gives $n$ nested intervals. These nested intervals are the focal elements and each interval is assigned the bpa $1 / n$. In all the proposed methods we also consider a triangular fuzzy number with the [min, max] as support and the most likely value as core. We then discretize the fuzzy number by considering finite number of alpha-cuts. That gives a family of nested intervals. Considering these nested intervals as focal elements we assign bpa by different approaches.

The focal elements are given by the alpha-cut as ${ }^{\alpha} A_{i}=\left\{x: \mu_{A}(x) \geq \alpha_{i}\right\}=\left[{ }^{\alpha} A_{i_{\text {lower }}},{ }^{\alpha} A_{i_{\text {upper }}}\right],(i=1,2,3 \ldots, n)$,

with $\quad \alpha_{0}=1>\alpha_{1}>\alpha_{2}>\ldots>\alpha_{n}=0$, where $\alpha \in[0,1]$.

(I) In the first approach we first integrate the membership function between different alpha-cuts i.e.

$$
\int^{\alpha}{ }^{\alpha} A_{i_{\text {ilower }}} \mu_{(A)}(x) d x
$$

The using normalizing procedure the basic probability assignments associated to ${ }^{\alpha} A_{i}$ is defined as

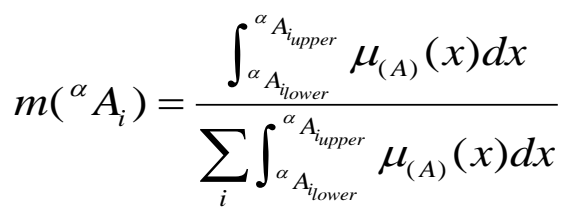

(II) In the second approach BPA is assigned as

$$
m\left({ }^{\alpha} A_{i}\right)=\frac{\alpha_{i-1}\left(1-\alpha_{i}\right)}{\sum_{i} \alpha_{i-1}\left(1-\alpha_{i}\right)}
$$

(III) In the third approach each $\alpha$-cut is considered as a confidence interval with level of confidence $1-\alpha$. Consequently using normalization procedure, the BPA for the focal element is assigned as

$$
m\left({ }^{\alpha} A_{i}\right)=\frac{1-\alpha_{i}}{\sum_{i}\left(1-\alpha_{i}\right)}
$$

\subsection{Numerical Example:}

To demonstrate and make use of the proposed methods we consider an example. We consider a the fuzzy number $A=[2$, $6,10]$ having membership function

$$
\mu_{A}(x)=\left\{\begin{array}{l}
\frac{x-2}{4}, 2 \leq x \leq 6 \\
\frac{10-x}{4}, 6 \leq x \leq 10
\end{array}\right.
$$

BPA is calculated using our proposed methods and which are given in the following table1, table 2 and table 3 respectively. Here we have considered alpha-cuts corresponding to $\alpha_{0}=1$, $\alpha_{1}=0.8, \alpha_{2}=0.6, \alpha_{3}=0.4, \alpha_{4}=0.2, \alpha_{5}=0.0$.

\begin{tabular}{|c|c|c|c|c|c|}
\hline $\begin{array}{c}\text { Focal } \\
\text { Elements }\end{array}$ & $\begin{array}{c}{[5.2,} \\
6.8]\end{array}$ & $\begin{array}{c}{[4.4,} \\
7.6]\end{array}$ & $\begin{array}{c}{[3.6,} \\
8.4]\end{array}$ & $\begin{array}{c}{[2.8,} \\
9.2]\end{array}$ & $\begin{array}{c}{[2,} \\
10]\end{array}$ \\
\hline BPA & 0.095 & 0.168 & 0.221 & 0.253 & 0.263 \\
\hline
\end{tabular}

Table 1: BPA obtained from fuzzy number using method (I)

\begin{tabular}{|c|c|c|c|c|c|}
\hline $\begin{array}{c}\text { Focal } \\
\text { Elements }\end{array}$ & $\begin{array}{c}{[5.2,} \\
6.8]\end{array}$ & $\begin{array}{c}{[4.4,} \\
7.6]\end{array}$ & $\begin{array}{c}{[3.6,} \\
8.4]\end{array}$ & $\begin{array}{c}{[2.8,} \\
9.2]\end{array}$ & {$[2,10]$} \\
\hline BPA & 0.1429 & 0.2286 & 0.2571 & 0.2286 & 0.1429 \\
\hline
\end{tabular}

Table 2: BPA obtained from fuzzy number using method (II)

\begin{tabular}{|c|c|c|c|c|c|}
\hline $\begin{array}{c}\text { Focal } \\
\text { Elements }\end{array}$ & $\begin{array}{c}{[5.2,} \\
6.8]\end{array}$ & $\begin{array}{c}{[4.4,} \\
7.6]\end{array}$ & $\begin{array}{c}{[3.6,} \\
8.4]\end{array}$ & $\begin{array}{c}{[2.8,} \\
9.2]\end{array}$ & $\begin{array}{c}{[2,} \\
10]\end{array}$ \\
\hline BPA & 0.067 & 0.133 & 0.200 & 0.267 & 0.333 \\
\hline
\end{tabular}

Table 3: BPA obtained from fuzzy number using method (III)

The total uncertainty is calculated in each of the above cases using (10).

\begin{tabular}{|c|c|c|c|}
\hline \multicolumn{4}{|c|}{ Total Uncertainty } \\
\hline Method I & Method II & Method III & $\begin{array}{c}\text { Existing } \\
\text { Method }\end{array}$ \\
\hline 1.9308 & 1.8224 & 1.9852 & 1.8335 \\
\hline
\end{tabular}

From the BPAs obtained from the three approaches we have calculated cumulative Plausibility and Belief measures. The figures below depict the bound of uncertainty.

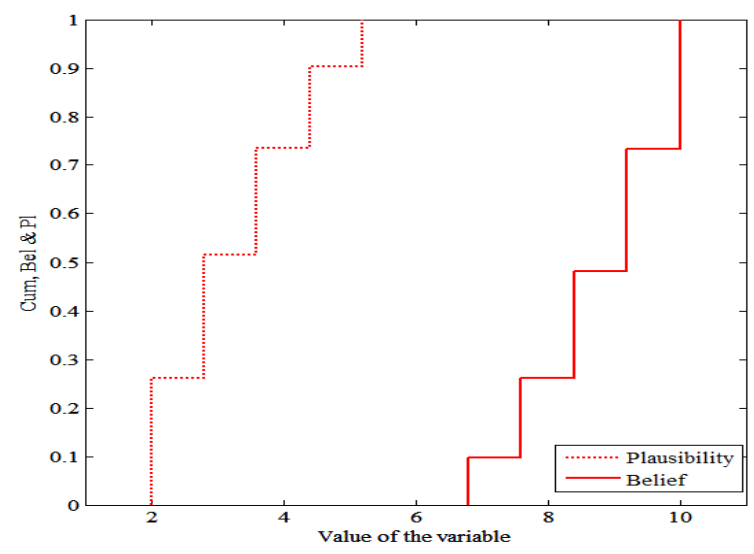

Plausibility and Belief obtained from Method-I 


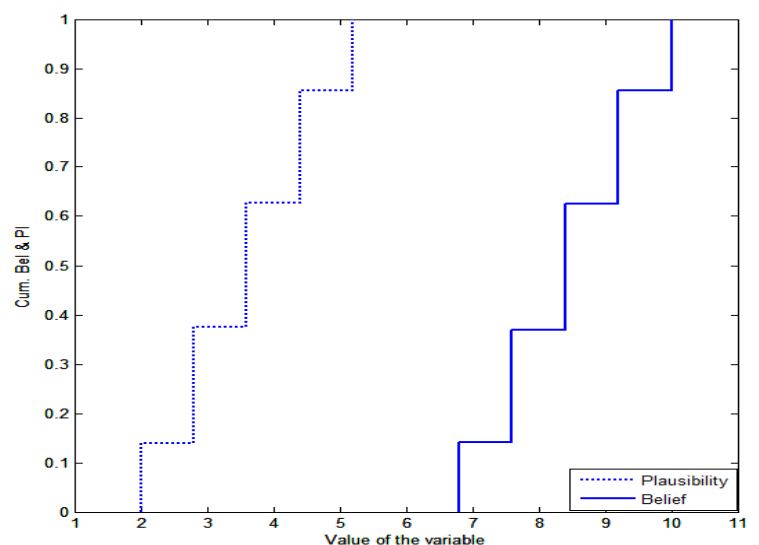

Plausibility and Belief obtained from Method-II

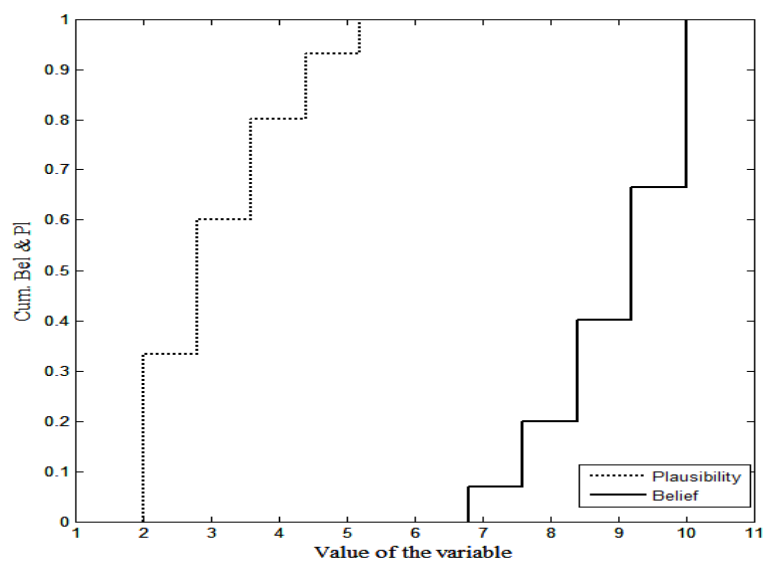

Plausibility and Belief obtained from Method-III

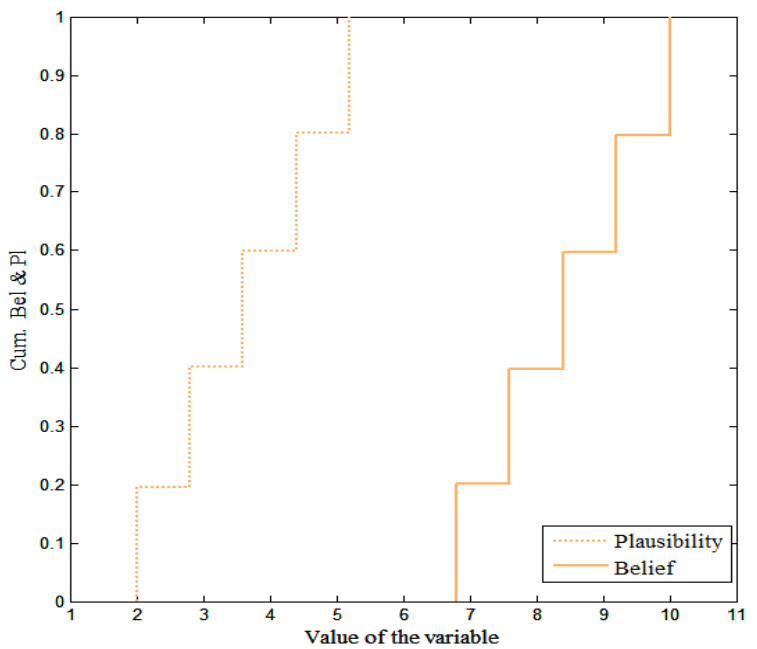

Plausibility and Belief obtained from the existing standard method

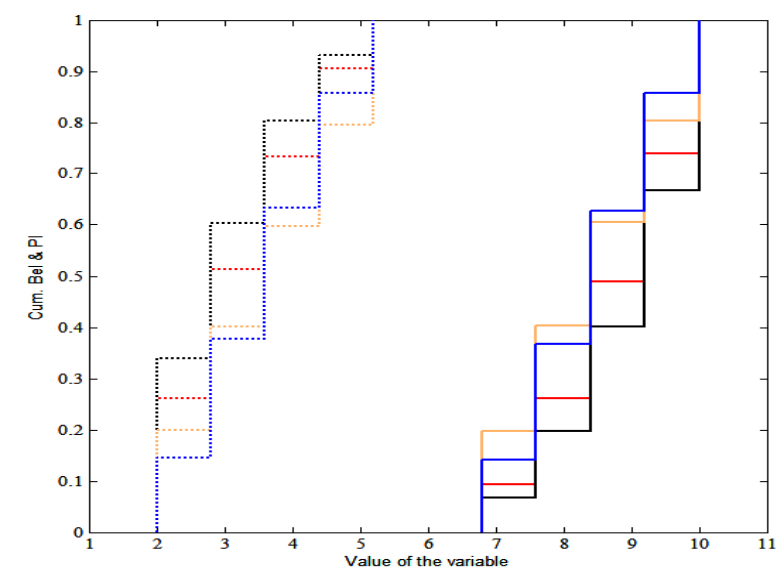

Superimposition of the measures obtained by four different approaches is depicted below:

Red: Method I

Blue: Method II

Black: Method III

Orange: Existing method

\section{Conclusion:}

Evidence Theory is a branch of mathematics that concerns the combination of empirical evidence in an individual's mind in order to construct a coherent picture of reality. It is an important tool of uncertainty modelling when both epistemic and aleatory uncertainties are present in the problem under consideration. In the absence of empirical data, experts in related fields provide necessary information. The fundamental and important object of this theory of evidence is the primitive function called basic probability assignment (bpa). The method of assigning bpas depends upon the problem under consideration and so it is an open issue. Various researchers have dealt with this issue. We have considered the situation when the only information available about the parameter under consideration is minimum value, maximum value and the most likely value. In general in such a situation a triangular fuzzy set (or a possibility distribution as the case may be) is considered with support as the interval [min, max] and the most likely value as the core. There is a standard procedure of obtaining focal elements from a fuzzy number and assigning bpas. However we feel that as the fuzzy number is obtained only from the information of the minimum, maximum and the most likely value, the existing procedure of assigning bpas may not be always reasonable. We have proposed three ways of assigning bpas in such situation. The three methods are compared with the existing standard method using a numerical example. We have also calculated total uncertainty in each of the cases. Total uncertain is seen least in Method II. That is also evident from the figure showing the superimposition of all the methods. We do not claim any method to be best. It all depends upon the underlying problem. We should clearly understand what the uncertain parameter actually represents and should know the nature of the uncertainty involved with the parameter before applying one or the other method of assigning bpa. 


\section{Acknowledgement}

The work done in this paper is under a research project funded by Board of Research in Nuclear Sciences, Department of Atomic Energy, Govt. of India.

\section{References}

[1] G.J.Klir and B.Yuan, Fuzzy sets and Fuzzy Logic Theory and Applications, Upper Saddle River, NJ Prentice -Hall, 1995.

[2] G. Shafer, A Mathematical theory of evidence, Princeton University Press, 1976.

[3] P. Dutta, H. Boruah., T. Ali Fuzzy arithmetic with and without using $\alpha$-cut method: a comparative study, International Journal of Latest Trends in Computing, 2011, 2(1): 99-108.

[4] S. P. Kurkowska, P. KOstka, A. Gertych, E. Straszecka and J. Straszecka, Basic Probability Assignment calculation in Diagnosis Support, European Symposium on intelligent Technique (ESIT), Aachen, Germany, 2000, pp.-92-94.

[5] Z. Yibing, L. Linhui, G. Lie, Z. Mingheng, New Initialization Method of Basic Probability Assignment and Application in Cross-country Environment Perception, International Conference on Intelligent System Design and Engineering Application,2010, pp.997-1001.

[6] Z. Zuo, Y. Xu and G. Chen, A New Method of Obtaining BPA and Application to the Bearing Fault Diagnosis of Wind Turbine, Proceedings of the 2009 International Symposium on Information Processing (ISIP'09), Huangshan, P. R. China, 2009, pp. 368-371.

[7] W. Jiang, Y. Deng, and J. Peng, A New Method to Determine BPA in Evidence Theory, Journal of Computers, Vol. 6, No. 6, 2011, pp.-1162-1167. 